\title{
Ultrafast, Self-Propelled Particles
}

\author{
New "Marangoni surfers" that whizz along at 10,000 body lengths per \\ second offer new insight into active matter propelled by surface-tension \\ gradients.
}

By Erika K. Carlson

$\square$ articles that propel themselves along a fluid surface-tension gradient that they generate themselves are called Marangoni surfers. To understand the dynamics of this form of "active matter," researchers have generally focused on how macroscopic Marangoni surfers induce surface-tension gradients by affecting either temperature or surfactant concentrations. Now, Kilian Dietrich of the Swiss Federal Institute of Technology (ETH) in Zurich and colleagues have investigated how both of these effects combine at the microscale, deriving a more comprehensive understanding of the factors that control the particles' speeds [1]. In the process, they created micrometer-scale surfers that can race along at up to a few centimeters-or about 10,000 body lengths-per second.

The Marangoni surfers that the team created are thermally activated, meaning the surface-tension gradients that drive them are created by nonuniform fluid temperatures. Each

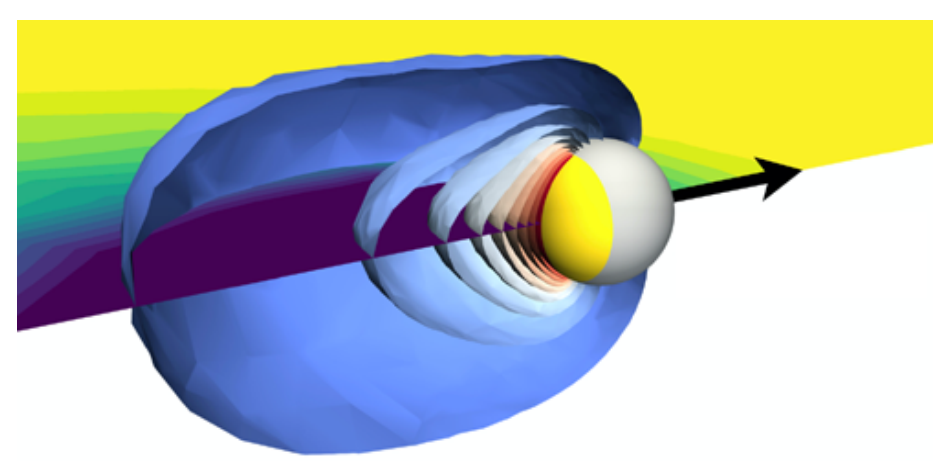

particle is a silica sphere, one-half of which is coated with a thin layer of gold. When the researchers shone a laser onto the particle, the gold-covered side warmed more than the bare silica side. The higher temperature lowered the surface tension of the fluid on the gold side, propelling the particle in the opposite direction.

Though the temperature gradient is what propels the particle, Dietrich and colleagues found that surfactant-concentration gradients oppose its motion once the particle is underway. As it travels, the Marangoni surfer leaves a wake in which the surfactant concentration is lower-and surface tension is therefore higher. Ultimately, the competing effects of the two opposing gradients determine the speed of the particle. The researchers anticipate that improvements in propulsion control emerging from this understanding will allow new routes to manipulate active matter systems at fluid interfaces.

Erika K. Carlson is a Corresponding Editor for Physics based in New York City.

\section{REFERENCES}

1. K. Dietrich et al., "Microscale Marangoni surfers," Phys. Rev. Lett. 125, 098001 (2020).

Credit: N. Jaensson/ETH Zurich 